\title{
Stem Cell Therapy in Acute Coronary Syndrome
}

\section{Dr. Rajeeb Rajbhandarl*}

Acule coronary syndrome and congestive heart failure are still among challenging problems in the field of cardiovascular medicine despite many advances in the field. Stem cell therapy has come as a new hope and a promise for the hopeless.

While preliminary data have supported the clinical of stem cell therapy to regenerate ischemic tissue in myocardium many issues still need to be resolved before this reaches to clinical practice. Safely and feasibility of different modalities of treatment are yet to be determined.

Cardiac myocyte lose the ability to divide after birth thus after myocardial infaretion there is irreversible damage to the heart. Till date reperfusion is the standard mode of treatment which is not very rewarding if it is done late. Use of stem cells for myocardial regeneration has been subject of recent interest and investigations.

Stem cells are defined as cells that have clonogenic capabilities and that differentiate into multiple cell lineages. When a stem cell divides each new cell either remain a stem cell or become a more specialized cell. The characteristic feature of the stem cell which distinguish them from other cells are their ability to renew themselves for long periods through cell division their capability to become cells with special function such as myocyte or endothelial cells.

Two kind of stem cells are used for the purpose: embryonic stem cells and adult stem cells. The adult and embryonic stem cell may be able to replace damaged heart muscle and establish and grow new blood vessel to supply them, Embryonic stem cells as their name suggests are derived from embryos. Embryonic cells are most primitive of all populations of stem cells, Adult stem cell is an undifferentiated cell found among differentiated cells in tissue organ. 
Human embryomie and adult stem cells each have advantages and disadvantages Regarding potential use of cell based regenerate therapies. Embryonic stem cell become all cell types of the body because they are pluripotent. Adult stem cells are generally limited to differentiation into different cell types of their tissue of origin. Transplanting fetal cardiomyocytes into a myocardial scar formed a new cardiac tissue and improved cardiac function Fetal cardiomyocytes may also contribute to The release of cardioprotective factors such as vascular endothelial growth factor through a paracrine effect that stimulate cell growth.

Results of various reports including BOOST trial TECAM pilot study TOP CARE- AMI trial and many more have investigated the role of stem cell as a therapy for cardiac regeneration in an effort to replace cardiomyocytes lost after ischemia. Bone marrow from adult contain three types of stem cell populations namely hemopoietic SC endothelial progeniter SC and mesenchymal stem cells.

The HSC produce all types of formed elements of blood and are isolated from either the bone marrow or blood. They are responsible for the constant renewal of blood cells Mesenchymal stem cells can be isolated from adult bone marrow and appear to have the potential to form multiple lineages of differentiation, MSC are a mixed cell population that generate bone cartilage fat etc.

Peripheral blood derived stem cell: cells isolated from adult human peripheral blood have shown to differentiated into non hematopoietic tissues, skeletal muscle unlike other types of muscle is able to regenerate and repair itself after injury due to the presence of immature satellite cells or myoblasts.

Of these skeletal myoblasts and bone marrow stem cells are preferred by most of the scientists. In one of the recent studies it has been shown that skeletal myoblast implantation was not associated with any adverse events or increased incidence of arrhythmia. In patients with previous myocardial infarction treatment with skeletal myoblast in conjunction with coronary artery bypass is safe and feasible and is associated with increased left ventricle function and improvement in viability. Many 
recent studies from Europe has shown that the autologous adult stem cells treated under appropriate conditions used for neovascularization and cardiac regeneration has shown a promising result and shown benefit in pilot clinical trials enrolling postinfarction patients with cornary artery disease.

Skeletal myoblast funtion as precursor cells that can undergo mitosis. Autologous myoblast are ideal for transplantation because readily available with a skeletal muscle biopsy specimen from the patient can be returned after vitro expansion and do not carry immunological and ethical concerns as human embryonic stem cell do. On the other hand endothelial progenitor cells are bone marrow residents that can be released into circulation after acute myocardial infarction and can produce neavascularization in the adult. No - need for immunosuppression.

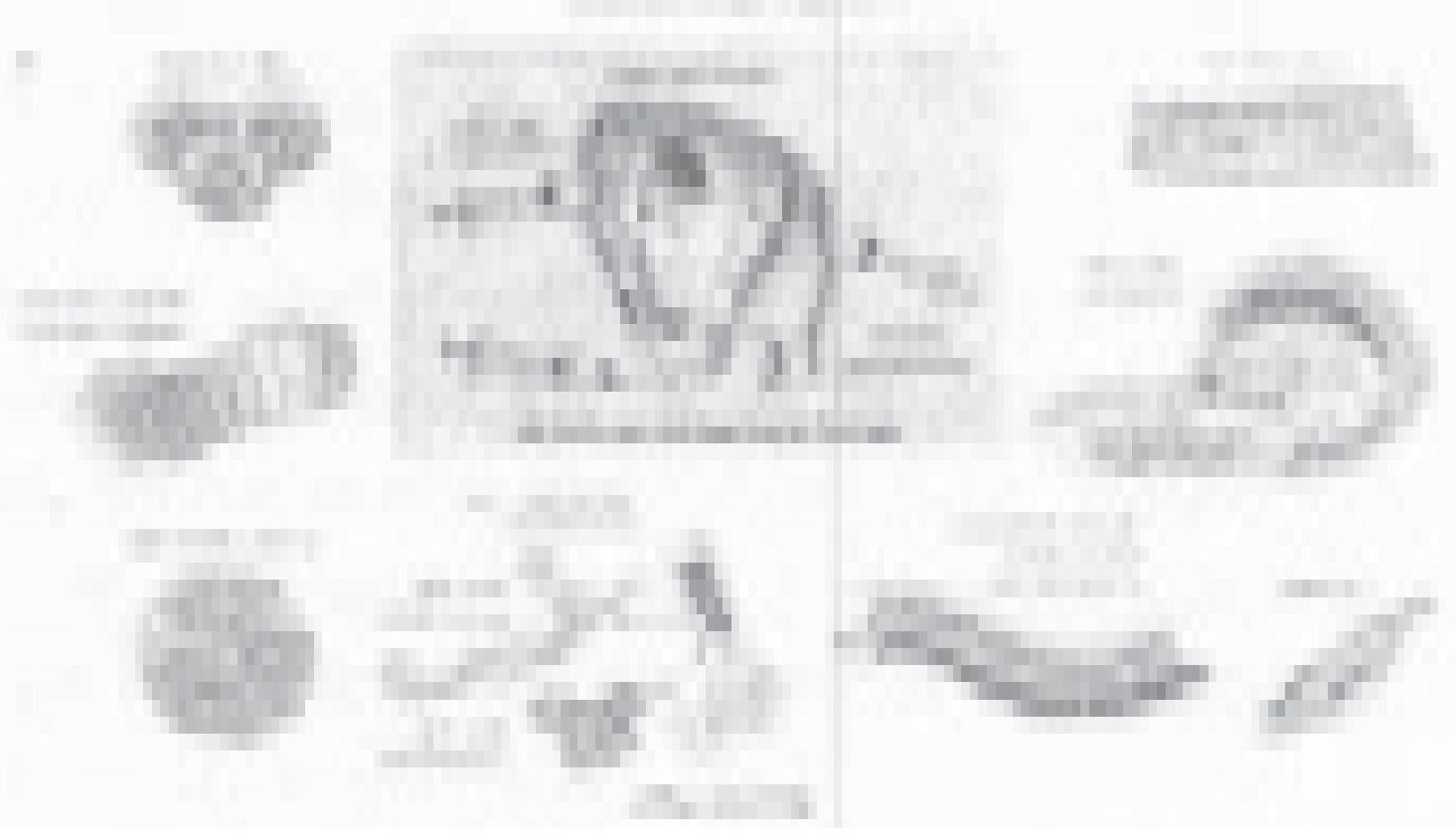


There are 3 or 4 routes of delivery, namely intramuscular injection intravenous injection intracoronary and transendocardial or trans-epicardial. Direct intramuscular into the damaged hear muscle has been used extensively in heart research. Advantage is direct loclization to the damaged are and disadvantage is the requirement of surgical procedure, IV administration of stem cell is an practical mode of delivery because it easy. Disadvantage is the length of time it takes for the cells to migrate to the site of the injury and the ability of the cell to survive. Tran-endocardial or trans-epicardial also referred to as myocardial, is also an invasive mode; There is need of surgery but requires fewer stem cells for transplantation. Intracoronary delivery is team cells via percutaneous transluminal coronary based catheter is superior to iv or intramuscular administration. This allows to home in and incorporate into the areas bordering the infarct zone in a homogenous manner and avoids the possibility of electrical instability and ventricular tachyarrythmia which is common in the case of intramuscular administration due to satellite like distribution.

Timing and number of cell transplantation appropriate for the patients is still a difficult decision. The damaged tissue releases some form of signal to attract the injected cells to an area of injury. Upon receiving the signal the stem cells reach the site of injury and begin the repair process. Fluoroscopy and cardiac MRI allows assessment of the size and location of the injection and extent of stem cell retention.

Incomplete integration of the stem cell into the myocardiumis a complication which leads to ineffective results and arrhythmia. Tumor formation and non cardiac tissue formation is another unwanted complication.

Although the stem cell implantation is still in its infancy and needs more large studies before it can be brought to clinic for routine use, we can hope this modality will come as a milestone in the management of cardiovascular diseases. 